\title{
Getting the elastic scattering length by observing inelastic collisions in ultracold metastable helium atoms
}

\author{
S. Seidelin, J. Viana Gomes*, R. Hoppeler, O. Sirjean, D. Boiron, A. Aspect and C. I. Westbrook \\ Laboratoire Charles Fabry de l'Institut d'Optique, UMR 8501 du CNRS, F-91403 Orsay Cedex, France
}

(Dated: October 31, 2018)

\begin{abstract}
We report an experiment measuring simultaneously the temperature and the flux of ions produced by a cloud of triplet metastable helium atoms at the Bose-Einstein critical temperature. The onset of condensation is revealed by a sharp increase of the ion flux during evaporative cooling. Combining our measurements with previous measurements of ionization in a pure BEC, we extract an improved value of the scattering length $a=11.3_{-1.0}^{+2.5} \mathrm{~nm}$. The analysis includes corrections taking into account the effect of atomic interactions on the critical temperature, and thus an independent measurement of the scattering length would allow a new test of these calculations.
\end{abstract}

Understanding and testing the role of interparticle interactions in dilute Bose-Einstein condensates (BEC) is an exciting area of current research. Although measurements of the interaction energy and the spectrum of excitations of a BEC have confirmed the validity of the Gross-Pitaevskii equation [1], there are still relatively few quantitative tests of other aspects, such as the effect of interactions on the value of the critical temperature $\left(T_{\mathrm{c}}\right)$ or the condensed fraction [2, 3]. The success in condensing metastable helium atoms $\left(\mathrm{He}^{*}\right)$ [4, 5], was greeted with interest in the community partly because the metastability offers new detection strategies unavailable with other species. To fully use these strategies however, we are still missing an accurate value of the s-wave scattering length $a$, the atomic parameter which determines all elastic scattering behavior at low energies. An accurate value of $a$ would also be useful to help clarify some puzzling results concerning measurements of $\mathrm{He}^{*}$ in the hydrodynamic regime, in which two different ways of measuring the elastic scattering rate appeared to be in contradiction [6]. Also, because He is a relatively simple atom, theoretical predictions of $a$ are already in a rather narrow range 7, 8] and these calculations should be tested.

A straightforward method to determine $a$ is to use ballistic expansion of a BEC to measure the chemical potential for a known atom number. This was done in Refs. [4, [5], but the measurements were limited by the calibration of the number of atoms, or equivalently the density of the sample. The reported values for $a$ are $20 \pm 10 \mathrm{~nm}$ and $16 \pm 8 \mathrm{~nm}$, respectively. A more recent estimate, limited by similar effects, is $a=10 \pm 5 \mathrm{~nm}[9]$. In this paper we report a new measurement of $a$ which makes extensive use of a unique feature of $\mathrm{He}^{*}$, spontaneous Penning ionization within the sample.

We exploit two specific situations in which the absolute atom number $N$ is simply related to $a$ and measured quantities: (i) for a pure BEC, the number is deduced directly from the chemical potential $\mu$ and $a$, (ii) for a cloud at the Bose-Einstein threshold it is simply related to the

\footnotetext{
*Permanent address: Departamento de Fisica, Universidade do
} Minho, Campus de Gualtar, 4710-057 Braga, Portugal critical temperature $T_{\mathrm{c}}$. Both $\mu$ and $T_{\mathrm{c}}$ are accurately deduced from time of flight (TOF) measurements. Comparison of ion rates from a pure BEC of known chemical potential and from a cloud at $T_{\mathrm{c}}$ allows us to extract $a$ and the ionization rate constants. The deduced value of $a$ is independent of the absolute ion detection efficiency, assuming that this (poorly known) efficiency is the same in the two measurements. The ion signal is also used in another novel way: Since it provides a real-time observation of the onset of BEC [10], we use it to reliably produce a cloud at the condensation threshold.

A dense cloud of $\mathrm{He}^{*}$ produces a steady flux of ions due to various ionization processes. Density losses due uniquely to ionizing collisions depend on the local density $n$ according to: $\left(\frac{d n}{d t}\right)_{\text {ionizing }}=-\frac{n}{\tau_{i}}-\beta n^{2}-L n^{3}$ with $\tau_{i}$ the lifetime due to ionizing collisions with the background gas and $\beta$ and $L$ the 2-body and 3-body ionization rate constants defined for a thermal cloud. The total ion rate from a thermal cloud is given by:

$$
\Phi=\frac{N}{\tau_{i}}+\frac{1}{2} \beta \int n^{2} d \mathbf{r}+\frac{1}{3} L \int n^{3} d \mathbf{r}
$$

The numerical factors reflect the fact that although 2 (3) atoms are lost in 2-body (3-body) collisions, only 1 ion is produced. Ionization measurements on a pure BEC were reported in [11], and, as $a$ was not precisely known, $\beta$ and $L$ were given in terms of $a$.

For a precise measurement of $a$, corrections due to interactions must be taken into account. In the mean field approach, the density is given by [1]:

$$
n(\mathbf{r})=\frac{1}{\lambda^{3}(T)} g_{3 / 2}\left[\exp \left(-\frac{1}{k_{B} T}(V(\mathbf{r})+2 g n(\mathbf{r})-\mu)\right)\right]
$$

with $\lambda(T)$ the thermal de Broglie wavelength, $T$ the temperature of the cloud, $k_{B}$ the Boltzmann constant, $V$ the trapping potential energy, $g=4 \pi \hbar^{2} a / m$ the interaction constant, $\mu$ the chemical potential and $g_{\alpha}(x)=\sum_{i=1}^{\infty} \frac{x^{i}}{i^{\alpha}}$.

The ion rate at the phase transition $\Phi_{c}$ can be derived from Eq. (2) by a first order perturbation theory similar to Ref. 12 but with a fixed temperature rather than a fixed atom number. We use the chemical potential of a 
gas in a harmonic potential at the BEC transition:

$$
\mu_{\mathrm{c}} / k_{B} T_{\mathrm{c}}=\frac{3}{2} \frac{\widetilde{\omega}}{\omega_{\mathrm{c}}}+4 g_{3 / 2}(1) \frac{a}{\lambda_{\mathrm{c}}}
$$

This gives:

$$
\begin{aligned}
\Phi_{\mathrm{c}}=\left(\frac{\omega_{\mathrm{c}}}{\bar{\omega}}\right)^{3} \times & {\left[\frac{1}{\tau_{i}}\left(1.20+2.48 \frac{\widetilde{\omega}}{\omega_{c}}+12.35 \frac{a}{\lambda_{\mathrm{c}}}\right)\right.} \\
& +\frac{\beta}{\lambda_{c}^{3}}\left(0.33+1.81 \frac{\widetilde{\omega}}{\omega_{c}}+6.75 \frac{a}{\lambda_{c}}\right) \\
& \left.+\frac{L}{\lambda_{c}^{6}}\left(0.22+2.21 \frac{\widetilde{\omega}}{\omega_{c}}+6.50 \frac{a}{\lambda_{c}}\right)\right]
\end{aligned}
$$

with $\widetilde{\omega}=\left(2 \omega_{\perp}+\omega_{\|}\right) / 3, \bar{\omega}=\left(\omega_{\|} \omega_{\perp}^{2}\right)^{1 / 3}, \omega_{\mathrm{c}}=k_{B} T_{\mathrm{c}} / \hbar$ and $\lambda_{\mathrm{c}}=\lambda\left(T_{\mathrm{c}}\right)$. The numerical values come from the calculation of arithmetic series and are independent of any parameters of the cloud. The terms proportional to $a / \lambda_{\mathrm{c}}$ in Eq. (4) account for the atomic interactions, while the corrections proportional to $\widetilde{\omega} / \omega_{\mathrm{c}}$ take into account the effect of finite sample size. For the typical parameters of our experiment we have $\left(T_{\mathrm{c}} \sim 2 \mu \mathrm{K}\right.$ and $\left.a=12 \mathrm{~nm}\right)$ $a / \lambda_{\mathrm{c}} \simeq \widetilde{\omega} / \omega_{\mathrm{c}} \simeq 0.02$ corresponding to an interaction correction of $20 \%, 40 \%$ and $60 \%$ in the three successive terms in Eq. (4). Even though the first order corrections are large, we find, using an approach similar to Ref. [13], that the second order corrections are negligible: $-4 \%$, $1.8 \%$ and $-3 \%$, respectively, with the above parameters. In our case, finite size corrections are always smaller than those due to interactions; since $a / \lambda_{\mathrm{c}} \simeq \widetilde{\omega} / \omega_{\mathrm{c}}$, the difference corresponds simply to the numerical constants in Eq. (4).

Our setup has been described in Ref. [11]. Briefly, we trap up to $2 \times 10^{8}$ atoms at $1 \mathrm{mK}$ in a Ioffe-Pritchard trap with a lifetime $(\tau)$ of $70 \mathrm{~s}$, and a lifetime due to ionizing collisions, $\left(\tau_{i}\right)$, estimated to be $>500 \mathrm{~s}$. In a typical run, forced evaporation for $30 \mathrm{~s}$ cools a cloud to a temperature near the phase transition. At this point, the rf-knife frequency is decreasing at a rate of $400 \mathrm{kHz} / \mathrm{s}$. We use a cloverleaf configuration with a bias field $B_{0}=300 \mathrm{mG}$. The axial and radial oscillation frequencies in the harmonic trapping potential are $\omega_{\|} / 2 \pi=47 \pm 3 \mathrm{~Hz}$ and $\omega_{\perp} / 2 \pi=1225 \pm 20 \mathrm{~Hz}$ respectively. A 2 -stage, single anode microchannel plate detector (MCP) is placed $5 \mathrm{~cm}$ below the trapping region. Two grids above the $\mathrm{MCP}$ allow us either to repel positive ions and detect only the $\mathrm{He}^{*}$ atoms, or to attract and detect positive ions produced in the trapped cloud. As explained in Ref. 11], to detect the ion flux, the MCP is used in counting mode, whereas we record the TOF signal at low gain (analog mode) to avoid saturation. As explained in Ref. [4], the TOF signal is due to atoms in the $m=0$ state which are insensitive to the magnetic field. However, atoms in magnetic field sensitive states are still present, and their trajectories are affected by uncontrolled residual fields. Therefore, during the time of flight, we apply a magnetic gradient in order to push these atoms away from the detector. The ratio between the detected atoms in the $m=0$ state and the initial number of atoms in the cloud is not well known 10, so we use the TOF only to get the temperature.

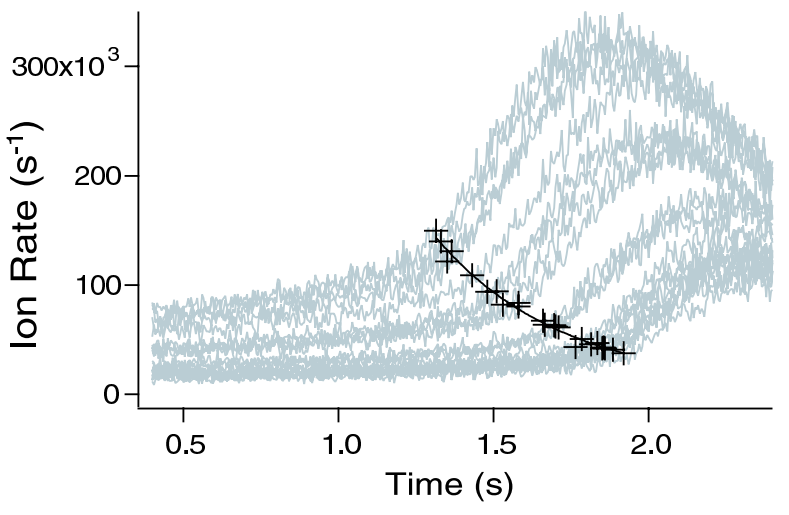

FIG. 1: Variation of the ion rate as the atomic cloud is cooled through the phase transition for various initial densities (gray curves). The rf-knife frequency at $t=0$ is $2 \mathrm{MHz}$. The sudden increase of the ion rate (crosses) occurs at the BEC transition. The solid line passing through the transition points constitutes our empirical relation, named threshold curve.

The crux of the experiment is to obtain a cloud of atoms at the phase transition. To identify the BEC threshold point, we monitor the ion signal. We have shown in Ref. 10] that the onset of BEC is heralded by a sudden increase of the ionization rate associated with the increased density of the condensate. More precisely, the BEC threshold corresponds to the rapid change in slope of the ion rate vs time, or the maximum of the 2nd derivative [14]. Figure 1 shows a series of such ionization rates during evaporation through the BEC transition. From these curves we can determine an empirical relation between the time of the onset of condensation and the ion rate preceding it. This relation stays valid only as long as we keep the same evaporation ramp and bias field. We will refer to this as the "threshold curve" in the following. Due to fluctuations of the bias field, we observe fluctuations of the time of BEC onset from run to run. These correspond to approximately $\pm 60 \mathrm{~ms}$ in time or $\pm 25 \mathrm{kHz}$ in frequency, a value which agrees with independent measurements of the fluctuations of the bias field.

Having established this relation, we can interrupt an evaporation sequence very close to the BEC threshold, and record the instantaneous ion rate as well as the corresponding TOF signal. We typically chose the interruption time for the evaporation ramp in advance, and then discarded the runs for which the interruption time was further than $\pm 60 \mathrm{~ms}$ away from the threshold curve.

We fit the associated TOF spectrum to determine the temperature (Fig. 21). We use Eq. (2) together with $\mu_{\mathrm{c}}$ given in Eq. (3) for the initial atomic density and then assume purely ballistic expansion of the cloud after the switching off of the trap. We shall refer to this fit as the Bose fit. The fits are weighted by an estimated uncertainty in each point of the TOF curve. To make this estimate, we chose a set of TOF spectra which appeared 


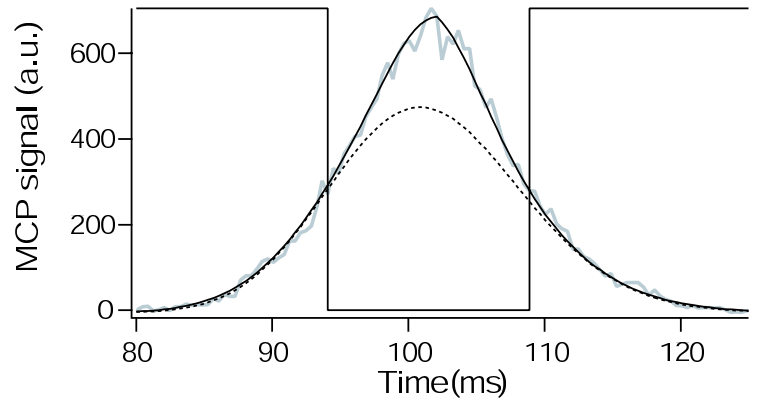

FIG. 2: Time of flight signal corresponding to a cloud released from the trap (at $t=0$ ) when its ion rate is on the threshold curve. We fit the data with an excluded window indicated by the vertical lines (width equal to the rms width of the TOF signal). A Gaussian function (dotted line) does not describe the central part of the data well, while the Bose function as defined in the text (solid line), does, indicating that the cloud is close to threshold.

to show no systematic deviation from their fits and used them to estimate the amplitude of the noise. This noise varies as the square root of the amplitude of the signal indicating that we are limited by the shot noise of the atom detection. Our procedure is only an approximate indicator of the error bars. The chi square per degree of freedom $\chi^{2}$ for the fits deduced in this way ranges from 0.8 to 3 . In addition, we first fit the TOF signal in its entirety and then exclude a successively larger window of the center of the spectrum out to the rms width of the spectrum. For all of our runs, we observe a variation of less than $5 \%$ and in most cases less than $3 \%$ of the fitted temperature as the excluded window is increased.

In Fig. 2] we show an example of a typical TOF spectrum and two fits to the data, using a Gaussian and the Bose function described above. Both fits shown use only the wings of the distribution. The ability of the Bose function to reproduce the center of the distribution without including it in the fit, unlike the Gaussian, confirms that the cloud is indeed close to the condensation threshold. In the following, we use the temperature given by the fit with an excluded window of half the rms width of the TOF signal in order to avoid the possibility of a small condensate component or other high density effects distorting our analysis.

Before plotting the ion rate as a function of the critical temperature, we must correct the observed temperature to account for the hydrodynamic expansion of the cloud (see [2] and references therein). This correction is done in the spirit of Ref. [15] which uses the Boltzmann equation approach to take into account the effects of collisions during the expansion of the cloud. The collision rate in Ref. 15] is calculated using a Gaussian density profile. We rather use the value calculated for an ideal Bose gas [16], which we have adapted to take interactions into account. This correction depends on the scattering length but the effect on the final value of $a$ is only of order
$0.3 \mathrm{~nm}$ for $a$ ranging from 10 to $14 \mathrm{~nm}$. We therefore simply assume $a=12 \mathrm{~nm}$ for this correction in the following. In fact, due to the additional anisotropy of the expanding cloud in the horizontal (detector) plane, the fitting function should be modified; but a simulation of this effect shows that the correction to the temperature is less than $0.1 \%$.

Finally, we correct the detected ion rate $\Phi_{\mathrm{c} \text {, det }}$ to account for the detection efficiency $\alpha$ such that $\Phi_{\mathrm{c}}=$ $\Phi_{c, \operatorname{det}} / \alpha$. It should be noted that the rate constants were obtained by ion rate measurements [1]. This means that they were also corrected: $\beta=\beta_{\operatorname{det}} / \alpha^{\prime}$ and $L=L_{\operatorname{det}} / \alpha^{\prime}$. Equation 4 shows that, as long as $\alpha=\alpha^{\prime}$, the detection efficiency cancels out and do not have any impact on the determination of $a$. We have checked experimentally that $\alpha=\alpha^{\prime}$. To allow comparison with figures in earlier publications, the ion rate and the theoretical curves shown in all the figures have been corrected using the same $\alpha$ as earlier, namely $\alpha=0.42[10,11]$.

The results of this analysis are plotted in Fig. 3 Curves corresponding to the expected variation for three values of the scattering length are also shown. We see that a large fraction of the data falls between $a=10$ and $14 \mathrm{~nm}$. The points at the highest temperatures however, show a tendency to fall near the theoretical curve for $a=10 \mathrm{~nm}$, while those at lower temperatures fall near $a=14 \mathrm{~nm}$. To analyze this tendency further we examine the TOF fits more closely using the $\chi^{2}$ value as an indicator of the confidence level of each measurement. A large $\chi^{2}$ could mean that the Bose function with $\mu$ imposed to $\mu_{\mathrm{c}}$ is not the right fit function, and therefore that the cloud is not sufficiently close to $T_{\mathrm{c}}$. As shown in Fig. 3. outliers tend to be correlated with a large $\chi^{2}$. Note however, that the remaining scatter in the data is too large to be accounted for by our a priori estimates of the uncertainties in our ion rate or temperature measurements, and we presume that it is due to fluctuations in the determination of the BEC threshold.

To determine the scattering length, we fit the black points in Fig. 3 with $a$ as a free parameter and using $\beta$ and $L$ parameterized by $a$ as in Ref. [11]. The fit gives (all points are given equal weight) $a=11.3 \mathrm{~nm}$. Our chief estimated uncertainty stems from the fact that our data show a systematic tendency to fall above the best fit at low temperature and below it at high temperature. To estimate this uncertainty, we fit the data (including gray points) separately for $T_{\mathrm{c}}$ below and above $2 \mu \mathrm{K}$. We find $a=13.8 \mathrm{~nm}$ for the low temperature data and $a=10.4 \mathrm{~nm}$ for the high temperature data. The uncertainties in the measurements of $\beta$ and $L$ also contribute to the uncertainty in Eq. (4) used for fitting. In fact, the uncertainties in $\beta$ and $L$ are highly correlated [11] and their contribution to the uncertainty is less than $0.5 \mathrm{~nm}$.

The error bars are obtained by summing quadratically the sources of uncertainties. Our final result for the scattering length is thus $a=11.3_{-1.0}^{+2.5} \mathrm{~nm}$. This result may be compared with the calculation of Ref. [8]. This work leads to $a=8 \mathrm{~nm}$ using the potential curves of Ref. [17]. 


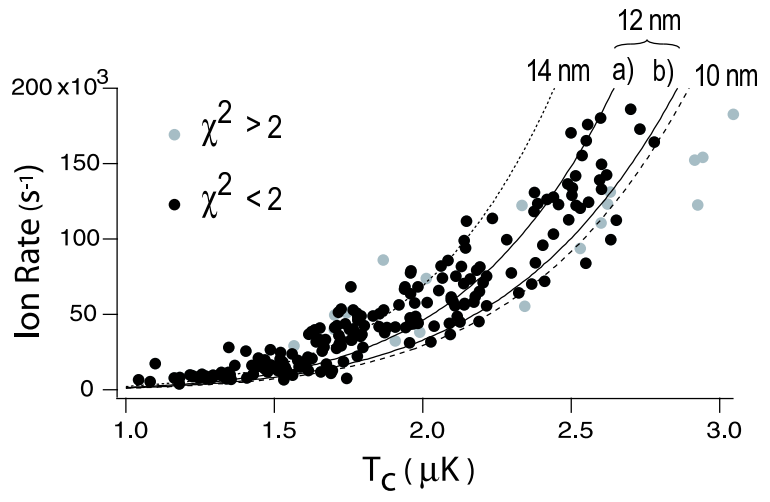

FIG. 3: Ion rate versus critical temperature. The points correspond to the results of 280 runs for which the ion rate was deemed sufficiently close to the condensation threshold. Gray indicates runs for which $\chi^{2}$ in the TOF fits was above 2. The dashed line is the theoretical estimate for $a=10 \mathrm{~nm}$, the dotted line for $a=14 \mathrm{~nm}$ (both including interaction corrections of Eq. (4). The two solid lines correspond to $a=12 \mathrm{~nm}$, a) with interactions and b) without interactions, and illustrate the size of their effect.

From Ref. 8] one also finds that a $0.5 \%$ shift of the repulsive part of that potential, would bring the theoretical value into agreement with our result. This $0.5 \%$ shift correspond to the estimated uncertainty in the potential of Ref. [17]. Another theoretical treatment [7] gives a scattering length between 8 and $12 \mathrm{~nm}$, also consistent with our results.

Our result also allows one to give values for the 2 and 3-body ionization rate constants. The error-bars of Ref. 11 are modified to take into account the uncertainty of $a$. The uncertainty in the ion detection efficiency also contributes to the uncertainty in the rate constants. As discussed in Ref. [11], we will assume $\alpha=0.42$ to get the central values of the rate constants. We will include a one-sided contribution to the errorbars to account for the possibility, also discussed in 11], that $\alpha$ could be a factor of 2 smaller. We finally get $\beta=0.9_{-0.8}^{+1.7} \times 10^{-14} \mathrm{~cm}^{3} / \mathrm{s}$ and $L=2.5_{-1.7}^{+4.5} \times 10^{-27} \mathrm{~cm}^{6} / \mathrm{s}$. The rate constants are in good agreement with theoretical predictions [8, 18].

As shown in Fig. 3. curves a) and b), our value of $a$ is significantly shifted by the non-ideal gas corrections of Eq. (4). Thus, when an independent measurement of the scattering length becomes available, our results can be used as a test of these corrections [19]. Note however, that corrections to the critical temperature beyond mean-field theory [20] are small when one parametrizes the critical point in terms of an average density [13]. But an examination of the critical density measured in a local way, by imaging the ions from a cloud for example, is sensitive to critical fluctuation phenomena which go beyond mean field theory similar to the homogenous case [20]. Thus, refinements of the ionization measurements described here promise to continue to provide new tests of BEC physics.

This work is supported by the EU under grants IST2001-38863 and HPRN-CT-2000-00125, and by the INTAS project 01-0855. SS acknowledges support from the Danish Research Agency and JVG from the Portuguese Foundation for Science and Technology (FCT).
[1] F. Dalfovo, S. Giorgini, L.P. Pitaevskii, and S. Stringari, Rev. Mod. Phys. 71, 463 (1999).

[2] F. Gerbier, J.H. Thywissen, S. Richard, M. Hugbart, P. Bouyer, and A. Aspect, Phys. Rev. Lett. 92, 030405 (2004)

[3] J.R. Ensher, D.S. Jin, M.R. Matthews, C.E. Wiemann, and E.A. Cornell, Phys. Rev. Lett. 77, 4984 (1996).

[4] A. Robert, O. Sirjean, A. Browaeys, J. Poupard, S. Nowak, D. Boiron, C.I. Westbrook, and A. Aspect, Science 292, 461 (2001).

[5] F. Pereira Dos Santos, J. Léonard, Junmin Wang, C.J. Barrelet, F. Perales, E. Rasel, C.S. Unnikrishnan, M. Leduc, and C. Cohen-Tannoudji, Phys. Rev. Lett. 86, 3459 (2001).

[6] M. Leduc, J. Léonard, F.P. Dos Santos, E. Jahier, S. Schwarz, and C. Cohen-Tannoudji, Acta Phys. Polon. B33, 2213 (2002).

[7] A.S. Dickinson, F.X. Gadéa, and T. Leininger, J. Phys. B. 37, 587 (2004).

[8] P. Leo, V. Venturi, I. Whittingham, and J. Babb, Phys. Rev. A 64, 042710 (2001).

[9] P.J.J. Tol, W. Hogervorst and W. Vassen, cond-mat/0312223

[10] S. Seidelin, O. Sirjean, J. Viana Gomes, D. Boiron, C.I.
Westbrook, and A. Aspect, J. Opt. B: Quantum Semiclass. Opt. 5, 112 (2003).

[11] O. Sirjean, S. Seidelin, J. Viana Gomes, D. Boiron, C.I. Westbrook, A. Aspect, and G.V. Shlyapnikov, Phys. Rev. Lett. 89, 220406 (2002).

[12] S. Giorgini, L. P. Pitaevskii, and S. Stringari, Phys. Rev. A 54, R4633 (1996).

[13] P. Arnold and B. Tomàs̆ik, Phys. Rev. A 64, 053609 (2001).

[14] O. Sirjean, Ph.D. Thesis, available at http://tel.ccsd.cnrs.fr/documents/archives0/00/00/30/88

[15] P. Pedri, D. Guéry-Odelin, and S. Stringari, Phys. Rev. A 68, 043608 (2003).

[16] G.M. Kavoulakis, C.J. Pethick, and H. Smith, Phys. Rev. A 61, 053603 (2000).

[17] J. Stärck and W. Meyer, Chem. Phys. Lett. 225, 229 (1994).

[18] G. V. Shlyapnikov, J.T.M. Walraven, U.M. Rahmanov, and M.W. Reynolds, Phys. Rev. Lett. 73, 3247 (1994); P. O. Fedichev, M. W. Reynolds, and G. V. Shlyapnikov, Phys. Rev. Lett. 77, 2921 (1996); P. F. Bedaque, E. Braaten, and H. W. Hammer, Phys. Rev. Lett. 85, 908 (2000).

[19] A measurement using photo-association spectroscopy is 
in progress at the ENS in Paris, M. Leduc, private communication.

[20] M. Holzmann, J.N. Fuchs, G. Baym, J.P. Blaizot, and
F. Laloë, cond-mat/0310460 N. Prokof'ev, O. Ruebenacker, and B. Svistunov, cond-mat/0401002 and references therein. 